MATEC Web of Conferences 31, 17002 (2015)

DOI: $10.1051 /$ matec conf/ 20153117002

(C) Owned by the authors, published by EDP Sciences, 2015

\title{
Design and Implementation of Browser based GPS/GPRS Vehicle Positioning and Tracking System
}

\author{
Keqiang Zhang ${ }^{1}$,Bingjie $\mathrm{Qu}^{2}$,Xiongfei $\mathrm{Chi}^{3}$ \\ Ordos Vocational College, 017000 \\ Ordos City, Mongolia, China
}

\begin{abstract}
This paper mainly describes a vehicle positioning and tracking system which is based on browser, GPS and GPRS. And this system takes advantage of Baidu Map as basic material to show vehicle status, which enables drivers and supervisor to monitor the vehicle's current and past positions. The vehicle's location data is got from satellites, and these data is sent to the central server through GPRS, the central server will store formatted data into the database after the data is parsed; Later, these data stored in the database will be used by web application and displayed on the map as markers. This paper also involves the implementation on mobile side, and this system used Baidu map JavaScript interface, Ajax, JSP and JSON to implement the vehicle positioning and tracking system.
\end{abstract}

\section{INTRODUCTION}

\subsection{SYSTEM REQUIREMENTS AND VALUE}

The system this paper describes will be applied into mining factory, there is several coaling stations, the coal will be carried away by vehicles. Sometimes, one coal station gathers many vehicles, and so that some of them often wait for a long time for shipment. At the meantime, some other coaling stations are not so busy, and have space to accept more vehicles. This system enable drivers and mining managers can monitor the status of vehicles from browser or mobile endpoint, this will avoid more vehicles gather in one coaling station, and it will be a efficient management means for modern mining factory and which enables mining manager's management be more efficient and ensure the production be under better management with high performance.

\subsection{DESIGN OF SYSTEM ARCHITECTRE}

This system consists of three parties, which are GPS/GRPS devices, central communication servers and the application based on browser(including the application of mobile), it integrates the main modern communication and internet technologies. Due to this system is built based on browser application, so that whatever drivers or the manager of mining factory can access this system from browser and mobile. The architecture diagram as following figure 1 .

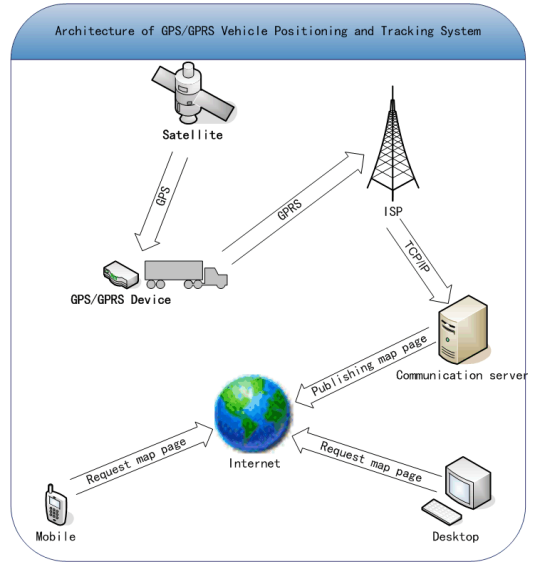

Fig 1: Vehicle positioning and tracking of GPS/GPRS based on mobile and browser

The current positioning of vehicles is got from GPS/GPS device which is fixed within the vehicle, after that,the data will be sent to central communication server which is in charge of receiving and parsing positioning data, then those data will be stored into the mongodb in a json format. These data will be searched by map generating module, which uses these data to show the position of vehicle on the map, and display the position of vehicle through http application.

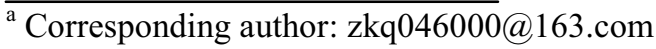




\section{TECHNOLOGIES USED IN THIS SYETEM}

\subsection{GPS}

GPS(Global Positioning System, GPS), which was developed in 1970 to overcome the limitations of previous navigation systems, integrating ideas from several predecessors, including a number of classified engineering design studies. GPS was created and realized by the U.S. Department of Defense (DoD) and was originally run with multiple satellites. It became fully operational . Bradford Parkinson, Roger L. Easton, and Ivan A. Getting are credited with inventing it. GPS equipment terminal can receive the positioning signal of more than three of the GPS satellite and can get high precision, global coverage, allweather speed, longitude and latitude, time, direction of geographic information, The Location positioning error in the range of 15 meters, Because GPS has wide coverage, allweather, high precision of a variety of advantages, it has been widely used in various industries worldwide in the field of. The vehicle location real-time monitoring system will become the most rapidly developing application market and promising. The GPS system includes three parts: First, a part of space - GPS satellite constellation, Second, ground control part- Ground monitoring system; Third, the user equipment Part-GPS signal receiver; The two have function independently, at the same time is also organically with integral whole.

\subsection{GPRS}

General packet radio service (GPRS) is a packet oriented mobile data service on the $2 \mathrm{G}$ and $3 \mathrm{G}$ cellular communication system's global system for mobile communications (GSM). A GPRS connection is established by reference to its access point name (APN). And for Internet communication services such as email and World Wide Web access. In order to set up a GPRS connection for a wireless modem, a user must specify an APN, optionally a user name and password, and very rarely an IP address, all provided by the network operator. Because GPRS is a group data data network, it supports TCP/IP, X.25 and so on.

\section{SYSTEM DETAIL DESIGN}

\subsection{SOFTWARES}

According to the architecture and functionality of the system, central communication server needs following softwares:
- Linux Ubuntu 12.0.4 LTS Operating System

- Mongodb 2.6.6 Software

- Tomcat 7.0 An application software which provides extra http service

\subsection{DETAILED DESIGN}

From the view of application itself, the central communication server mainly consists of data receiving module and map generating module. The data receiving module, as metioned before, is used to receive the data which is sent from GPS/GPRS devices, map generating module will use these data to generate map and provide http service for outside clients, see following detailed data flow by figure 2 :



Fig.2: System data flow

\subsubsection{DATABASE DESIGN}

The backend database is MongoDB because it's a very popular No-sql database currently, and is widely used by multiple big enterprise company. It is a document oriented database which format is JSON, it differentiates from the traditional database, MongoDB is more flexible. Every single document in the database represents the location of one vehicle at some time, it mainly consists of id of device, longitude, latitude, date time of data generation, also it support custom fields and which make the database be more flexible and extended easily. Following table 1 lists the definition of MongoDB document:

\{“id" : "k12345", "long":111.898989, "lat": 78.098789, “datetime":ISODate("2014-12-01T13:22:57Z",

"custom_fields": \{\}$)\}$ 
ICMEE 2015

Table 1 Definition of vehicle location data

\begin{tabular}{lll}
\hline \multicolumn{1}{c}{ Field } & \multicolumn{1}{c}{ Type } & \multicolumn{1}{c}{ Description } \\
\hline id & char & Required. Unique id of GPS/GPRS device \\
\hline long & Float with 14 points & Required. The longitude of vehicle \\
\hline lat & Float with 14 points & Required. The latitude of vehicle \\
\hline datetime & Time & Required. The timestamp of data generation \\
\hline \multirow{3}{*}{ custom_fields } & \multirow{2}{*}{ JSON } & Optional. Include possible and extendable data, such as the \\
& & speed, direction of vehicle, format is: custom_fields": ‘ “speed”: \\
\hline
\end{tabular}

\subsubsection{DATA RECEIVING MODULE}

The data receiving module, which is a service within the central communication server, and listening on port 9000 , is used to receive data sent from GPS/GPRS device and store data into the $\mathrm{db}$. The data sent to the central communication server through GPRS by TCP/IP interface, the server will parse these data and the its format looks like following: \$GPGGA, 013554.000,3936.927670,N,10948.95237E,1,5,2.42,1270.473,

$\mathrm{M},-28.022, \mathrm{M},{ }^{*} 45$ 。 The parsed data in the db looks like following:

\{“_id":"K12345", “long": 109.797816, "lat": 39.605589, \}

"datetime":

ISODate("2014-12-04T08:00:29+0000")\}。

Following is code segment for this module:

public void doGet(HttpServletRequest req,

HttpServletResponse res)

throws ServletException, IOException \{

String id = req.getParameter("id");

String long = req.getParameter("long");

String lat = req.getParameter("lat");

/** Store the data into the database*/

dbConnector.store(id, long, lat, datetime, $\mathrm{cf}$ );

\}

\subsubsection{MAP GENERATION MODULE}

The map generation module, a service within central communication server, is listening on port 80 . This module will search the location data of vehicle from the database, and used to generate map after the module is called by clients browser or mobile, it will provide http service for outside clients, such as mobile application, phone and computer, so that the vehicle tracking is finished. The benefit from using browser is that the http service can be used directly by not only computer browser but also mobile application, without any change for specific platform, In addition, this module also designed to provide following functionalities:

- The map generation module can read data from the database and mark the location on the map, also, it will show the total number of vehicles of every coaling station in a specific radius.
- Based on the center of coaling station, design the solution in 300 meters to show the vehicle.

Following will introduce the detailed design about how to generate on map for vehicles.

- Initialize amp

This system is using Baidu map as basic map material to show the location of vehicle on the map, besides of the rich API Baidu provides, and it provides the functionality of marker on the map. The map generation modules generate a JSP page to show the map and the markers of vehicle. In this JSP, will include the header of Baidu map $<\% @$ page language="java" content Type $="$ text $/ \mathrm{html} ; \%>$, to initialize the map, such as involving

$<$ script type="text/javascript" $\mathrm{src}=$ "http://api.map.baidu.com/api? $\mathrm{v}=2.0 \& \mathrm{ak}=$ your

key" $><$ /script $>$ into the JSP, so that the entire JSP can call various Baidu api, map = new BMap.Map("allmap"); After that, a central location on map is needed, it looks like: map.cente AndZoom(new BMap.Point(109.797816,39.605589), 15);

- Get the map data

To get the location of vehicles from JSP page, the JSP page need query the database periodically, it will refresh the markers on the map to make the map is up-to-data with the latest location data of vehicles, it does not refresh entire JSP page with Ajax technology, following is segment with Ajax to get the location data:

\$.ajax $(\{$ url: http://192.168.1.100/v1/geo.json

\}).done(function( data ) \{

$/ /$ Processing data.

//See following description about data structure \}$)$;

The response data for above request is looks like following: \{vehicles: [ \{ id: "K12345",long: 109.797816, lat: 39.605589, datetime: 2014-12-04T08:00:29+0000 \}]\}

\subsection{ACCESS THE SYSTEM BY BROWSER}

On the map page, the JSON data will be parsed by JavaScript, so that the page gets the longitude and latitude of vehicle, with 
the map marker, such as: var point $=$ new BMap.Point(long, lat);So that the location of vehicle can be displayed on the map, On the internet, there is unique address for each resource, it is named URL (Uniform Resource Locator), it's indicator of www, it can be accessed by http protocol, the URL of map looks like: http://192.169.1.110/v1/geo.json, the location can be shown for the specific vehicle on the map, 如 : http://192.169.1.110/v1/geo.json?id=K12345 。 Through the browser, mining manager can view the status of all vehicles and monitor them reat time.

\subsection{IMPLEMENT ON COMPUTER AND MOBILE}

With the development of mobile communication, in particular, the wide cover of smart phone which has the functionality of GPS, it becomes to true to monitor vehicle on mobile. In this system, because the location is shown as map, and which is abide of http protocol, It's easily integrated into Apple iOS application without any change. The overall flow chart of client terminal application, as shown in figure 3, Start the program and enter the system login interface. If there is no account password to user registration, After a successful login to enter the home page, Vehicle selection, extraction of latitude and longitude, the map display real-time location and the recent historical track playback function.

following is core code segment for iOS application:

NSString *ourPath = @"http://192.169.1.110/v1/geo.json"; NSURL *ourURL = [NSURL URLWithString:ourPath];

[ourApplication openURL:ourURL];

When the application is opened on mobile, the map will be open automatically, and shown on the mobile side, so that all the location of vehicles can be viewed from Apple iOS devlices.

Following Figure 4 is sample on Apple iSO device:

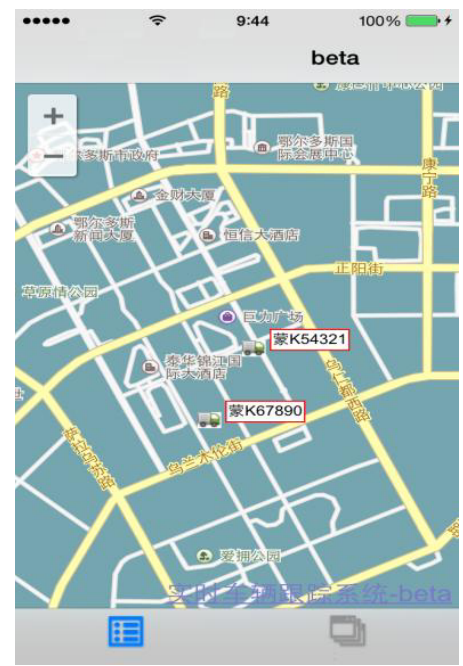

Fig.4: Integration of monitoring system on mobile

\section{CONCLUSION}

This paper takes advantage of modern communication and information technology to design and implement a vehicle positioning and tracking system, which can be used on both browser and mobile. The big character of this system is one system can be used by different platforms, as well as Android devices. It has the ability of cross-platform, energy-saving and high performance management.

\section{ACKNOWLEDGEMENTS}

The research work was supported by Application Technology Research and Development Foundation of Inner Mongolia Autonomous of China under Grant No. 20120304

Much thanks to my family members, dear colleagues when this paper is to be finished, all of them give me much favor and great help when I do research. 


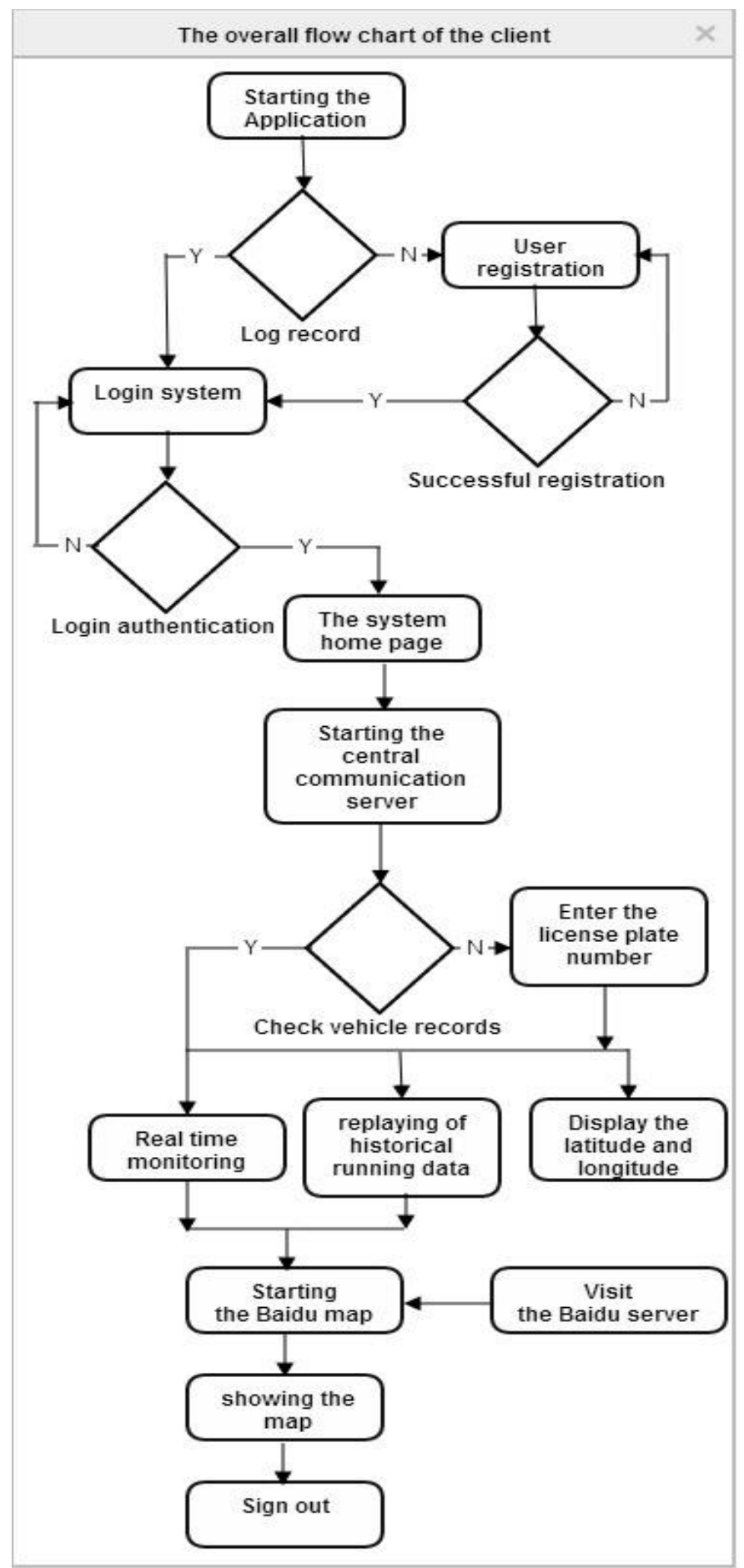

Fig.3:The overall flow chart of the client
3. LiuDajie. The global positioning system (GPS) theory and data processing[M].Shanghai:Tongji University press,2007.

4. ZhouZhongmo,YiJiejun, Principle and application of GPS satellite[M]. The publishing house of Surveying and mapping.1992.

5. SaShixuan,WangShan. Introduction to database systems (Third Edition) [M]. Beijing:Higher Education Press,2000.

6. Kaplan, Dean. iPhone 5 Application Sketch Book: For iPhone 5s, iPhone 5c and Earlier Models Running IOS 7 Apps. 2013-12

7. YangShaobo. J2EE Web core technology.Tsinghua University press.2011-01.

8. YuanAncun. The global positioning system (GPS)principle and Application[M].Dalian; Dalian Maritime University press. 1999:70-162.

9. Yong-Hua Cheng,Wen-Kuang Kuo,Szu-Lin Su. An Android System Design and Implementation for Telematics Services. 2010 IEEE International Conference on Intelligent Computing and Intelligent Systems (ICIS), 2010,(2):206-210.

10. Shin,WookKiyomoto, ShinsakuFukushima, Kazuhide Tanaka, Toshiaki.A Formal Model to Analyze the Permission Authorization and Enforcement in the Android Fraxnework[C] .IEEE International Conference on Social Computing (SocialCom 2010) 2011.1.

11. HanMin,Feng Hao,Geographic information data exchange method research based on JSON. Science of Surveying and mapping[J],2010, The thirty-fifth volume first issue: 155161

12. Huchengwei. Mobile webserver to the Android platform[J]. 2010 ISECS. International Colloquium on Computing, Communication, Control, and Management(CCCM)

13. Fawcett,ed. Adaptive Routing for Road Traffic -An integrated system uses road congestion information to guide routing. IEEE computer graphics and applications, 2000(3): 47-52 .

14. Cong Fu, Yan Wang, Yanqing $\mathrm{Xu}$, et al. The logistics network system based on the Google Maps API. in: Proceedings of 2010 International Conference on Logistics Systems and Intelligent Management. Harbin, China: IEEE Computer Society Press, 2010. 1486 1489

\section{REFERENCES}

1. QiuZhihe, WangWanyi.Principle and application of GPS[M]. Publishing House of electronics industry,2002.

2. LiuWei. The global positioning system (GPS) data processing[M]. Hunan; Publishing House of Elephant.2008. 